\title{
Phylogenetic clustering increases with elevation for microbes
}

\author{
Jianjun Wang, ${ }^{1,2}$ Janne Soininen, ${ }^{3}$ Jizheng $\mathrm{He}^{2}$ and \\ Ji Shen ${ }^{1 *}$ \\ ${ }^{1}$ State Key Laboratory of Lake Science and \\ Environment, Nanjing Institute of Geography and \\ Limnology, Chinese Academy of Sciences, Nanjing \\ 210008 China. \\ 2State Key Laboratory of Urban and Regional Ecology, \\ Research Center for Eco-Environmental Sciences, \\ Chinese Academy of Sciences, Beijing 100085 China. \\ ${ }^{3}$ Department of Environmental Sciences, PO Box 65, \\ FIN-00014, University of Helsinki, Finland.
}

\section{Summary}

Although phylogenetic approaches are useful for providing insights into the processes underlying biodiversity patterns, the studies of microbial phylogenetic relatedness are rare, especially for elevational gradients. Using high-throughput pyrosequencing, we examined the biodiversity patterns for biofilm bacterial communities that were scraped from stream stones along an elevational gradient from 1820 to $4050 \mathrm{~m}$ in China. The patterns of bacterial species richness and phylogenetic diversity were hollow towards higher elevations. The bacterial communities consisted of closer relatives than expected and displayed increasing terminal phylogenetic clustering towards mountain top. The increasing phylogenetic clustering with elevation contrasts reports for macroorganisms that revealed phylogenetic overdispersion at low or intermediate elevations. Because water temperature showed the strongest correlation with phylogenetic relatedness $\left(r^{2}=0.516\right)$, the elevational pattern in the bacterial phylogenetic structure indicated that environmental filtering possibly due to lower temperature or more frequent temperature fluctuations increased towards higher elevations. Evidence supporting the environmental filtering on bacteria was also reflected by the orderly succession in the relative abundance of different bacterial phyla along the elevational gradient and in the high evenness of bacterial taxa at higher elevations. Overall,

Received 19 August, 2011; revised 7 December, 2011; accepted 15 December, 2011. *For correspondence. E-mail jishen@ niglas.ac.cn; Tel. (+86) 258688 2005; Fax (+86) 2557713063. our results indicated that ecological processes possibly related to temperature may play a dominant role in structuring bacterial biodiversity along the elevational gradient.

\section{Introduction}

Since the development of community phylogenetics, the use of the phylogenetic framework to study the forces underlying biodiversity patterns has greatly increased in plants and animals (Webb, 2000; Emerson and Gillespie, 2008; Cavender-Bares et al., 2009; Vamosi et al., 2009), as well as for microbes (e.g. Martin, 2002; Horner-Devine and Bohannan, 2006; Bryant et al., 2008; Auguet et al., 2010; Jones and Hallin, 2010). This framework uses the information on phylogenetic relatedness that was derived from phylogenetic trees and assumes that phylogenetic relatedness is correlated with ecological similarity. By comparing the phylogenetic structure observed for a natural community and the communities randomly assembled from a larger species pool, the relative importance of evolutionary and ecological forces in shaping the community can be separated (Webb et al., 2002; Kembel and Hubbell, 2006; Cavender-Bares et al., 2009; Kembel, 2009). Phylogenetic approaches have been implemented to examine several ecological topics, such as the spatial distribution of phylogenetic diversity (Morlon et al., 2010), the succession of invasive species in a community (Cadotte et al., 2009) and patterns of elevational diversity (Bryant et al., 2008; Graham et al., 2009).

Mountainsides often provide a promising natural laboratory for studies of biodiversity and biogeography (Lomolino, 2001; Rahbek, 2005; Reche et al., 2005; Grytnes and McCain, 2007). Despite growing interest in the phylogenetic framework, most studies on elevational diversity have been conducted using traditional taxonomic richness (Rahbek, 2005; Grytnes and McCain, 2007), and a few studies have addressed phylogenetic diversity (e.g. Bryant et al., 2008; Vamosi and Queenborough, 2010). This is a drawback because accounting for phylogenetic relatedness may provide additional insight into the patterns of community structure along an elevational gradient or the processes that underlie these patterns (CavenderBares et al., 2009; Kembel, 2009). Until recently, only a few studies tested these patterns, but with diverse organism groups, such as plants (Bryant et al., 2008; Kluge and 
Kessler, 2010), hummingbirds (Graham et al., 2009), ants (Machac et al., 2010) and Acidobacteria (Bryant et al., 2008). In one of these studies, Graham and colleagues (2009) studied phylogenetic structure in tropical hummingbird communities along an elevation and found that communities were phylogenetically overdispersed (i.e. locally coexisting species were typically distant relatives) possibly due to competition at low elevations whereas communities were phylogenetically clustered (i.e. coexistence of closely related species) due to environmental filtering at higher elevations.

Even though most published datasets regarding microbial diversity are molecular in nature, the phylogenetic relatedness of microbial communities has only been examined within the past few years, but with a considerable recent increase (Martin, 2002; Bohannan, 2003; Horner-Devine and Bohannan, 2006; Barberán and Casamayor, 2010; Jones and Hallin, 2010; Amaral-Zettler et al., 2011). Based on the taxonomic richness, four recent studies have documented contrasting elevational patterns for microbes - decreasing (Bryant et al., 2008), increasing (Wang et al., 2011) or unimodal (Singh et al., 2011) richness with increasing elevation, or no pattern (Fierer et al., 2011). To the best of our knowledge, only one study has examined the patterns in microbial phylogenetic structure with respect to elevations (Bryant et al., 2008). The elevational pattern of phylogenetic structure for Acidobacteria was different from the corresponding finding for angiosperms (Bryant et al., 2008).

Here, we investigated bacterial biodiversity along the elevations of $1820-4050 \mathrm{~m}$ in the biofilm of a stony stream in China. As an earlier analysis of these samples used denaturing gradient gel electrophoresis (DGGE) (Wang et al., 2011), we used here high-throughput pyrosequencing for employing the community phylogenetic tools. We described the bacterial community composition at the phylum level because it has been documented that species composition may strongly affect ecosystem functions (Downing and Leibold, 2002), and little information is available regarding the elevational patterns of aquatic bacterial communities in general. In addition, we studied elevational patterns in species richness, phylogenetic diversity and phylogenetic structure for the communities, and correlated these with measured environmental variables to reveal potential underlying factors.

\section{Results}

In total, more than 21 microbial phyla were detected from the biofilm in the studied stream. Among all of the phyla, Alphaproteobacteria were the most abundant at 15 sampling sites, and Cyanobacteria were the most abundant at five lower sites (Fig. 1). The relative abundance of some phyla, such as Bacteroidetes (Fig. 1B), Actinobacteria
(Fig. 1C) and Deltaproteobacteria (Fig. 1G), increased with elevation, while the relative abundance of the other phyla showed highly divergent elevational patterns (Fig. 1I-P). For example, the abundance of Alphaproteobacteria (Fig. 1I) and Cyanobacteria (Fig. 1J) tended to decrease with elevation. The redundancy analysis (RDA) analyses revealed that the elevation was the strongest factor $(P<0.01)$ that was correlated with the distribution of phyla (Fig. 2). Total nitrogen (TN) and latitude also showed marginally significant correlations with community composition ( $P=0.056$ and $P=0.058$ respectively), while the other factors were all non-significant when elevation was included in the analyses (Fig. 2).

Chao1-richness followed a hollow pattern $\left(r^{2}=0.51\right.$, $P<0.001)$ : the lowest richness was detected at approximately $2300 \mathrm{~m}$, and the highest richness was detected at approximately 3400 m (Fig. 3A). Accordingly, Faith's phylogenetic diversity (PD) correlated with Chao1-richness (Pearson's $r=0.942, P<0.001$ ), and also showed a hollow pattern $\left(r^{2}=0.58, P<0.001\right.$; Fig. $\left.3 \mathrm{~A}\right)$. Pielou's evenness of the taxonomic communities showed a unimodal pattern with elevation $\left(r^{2}=0.49, P<0.001\right.$; Fig. 3B $)$ that was consistent with the hollow pattern for the imbalance metric (Colless's index) for the phylogenetic trees $\left(r^{2}=0.62, P<0.001\right.$; Fig. 3B $)$.

However, compared with the richness patterns, the community structure showed differing distribution patterns along the elevational gradient. For instance, mean nearest taxon distance (MNTD) decreased linearly with increasing elevation $\left(r^{2}=0.49, P<0.001\right.$; Fig. $\left.3 C\right)$, which indicates stronger clustering at higher elevations. All of the standardized effect sizes of MNTD (ses.MNTD) that were obtained using the null model were significantly negative, which indicates that the bacterial communities had a tendency to be more phylogenetically clustered than expected by chance. Further, the standardized metric showed a pattern with elevation $\left(r^{2}=0.66\right.$, $P<0.001$; Fig. 3D) that was similar to that of MNTD, which indicates that the generated random effects have only minor influence on the elevational pattern of the phylogenetic structure.

When the phylum Proteobacteria was considered separately, the patterns in Chao1-richness, Faith's PD and Colless's index, and the patterns in community evenness were similar to those of the whole bacterial communities (hollow and unimodal respectively) (Fig. 4A and B). However, MNTD decreased with increasing elevation in a manner that followed the quadratic model $\left(r^{2}=0.46\right.$, $P<0.001$ ) (Fig. 4C). The standardized MNTD values of the Proteobacteria communities followed a decreasing pattern that was similar to that of MNTD $\left(r^{2}=0.66\right.$, $P<0.001$ ) (Fig. 4D).

The correlations between biodiversity and the environmental variables were examined by multiple ordinary least 

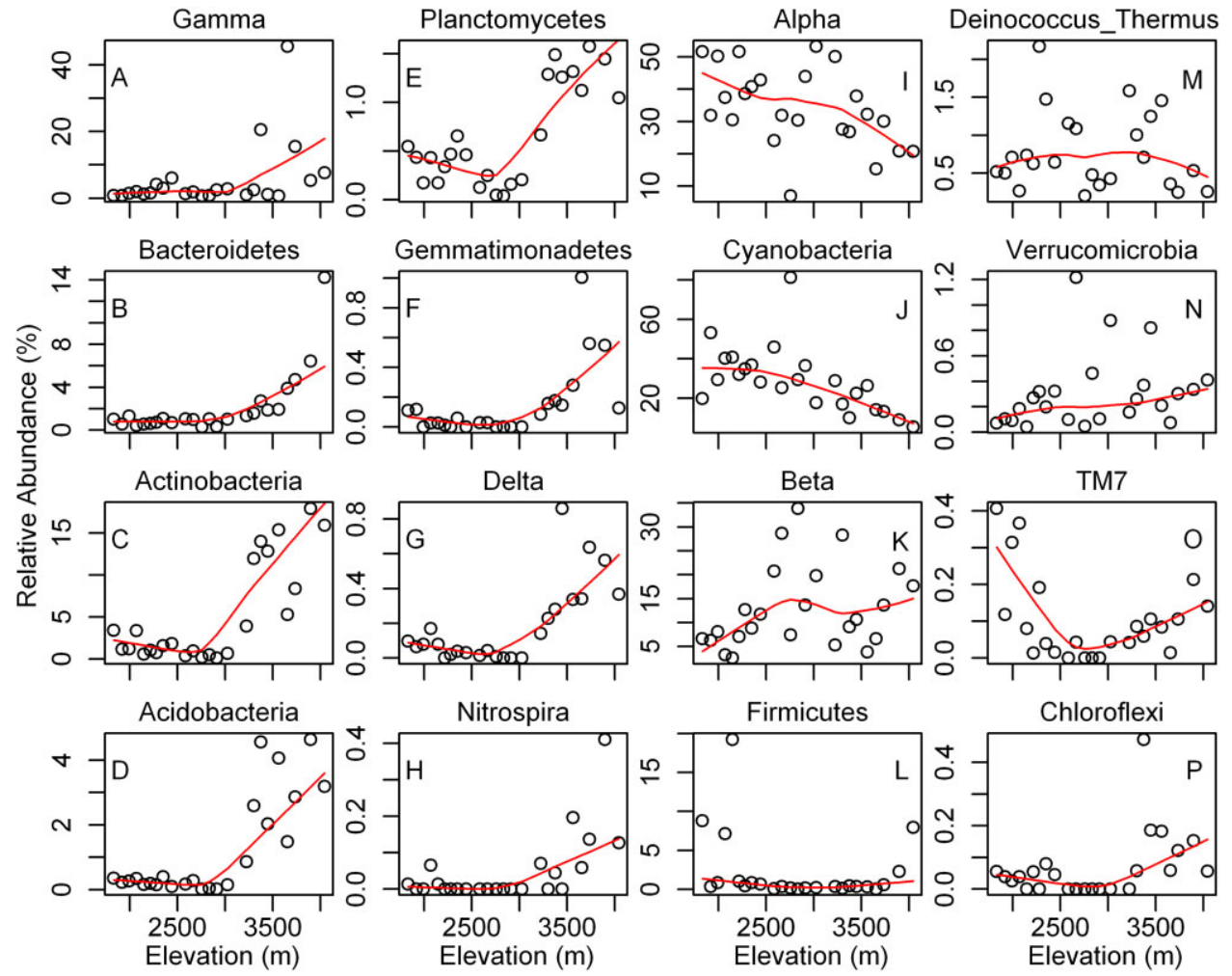

Fig. 1. The relative abundance of bacterial phyla along the elevational gradient. (A) Gammaproteobacteria, (B) Bacteoidetes, (C) Actinobacteria, (D) Acidobacteria, (E) Planctomycetes, (F) Gemmatimonadetes, (G) Deltaproteobacteria, (H) Nitrospira, (I) Alphaproteobacteria, (J) Cyanobacteria, (K) Betaproteobacteria, (L) Firmicutes, (M) Deinococcus, (N) Verrucomicrobia, (O) TM7 and (P) Chloroflexi. The trends along the elevation were indicated by solid lines using Locally Weighted Smooth Regression.

squares (OLS). The environmental variables had a high explanatory power (all $r^{2}>0.646$ ) for biodiversity even when the spatial effects were controlled for (Table 1). For the bacterial communities, the stream water temperature was the most important correlate for Chao1-richness, Faith's PD and MNTD (Table 1). For Proteobacteria, the stream water temperature showed the highest correlations with Chao1-richness and Faith's PD (Table 1)

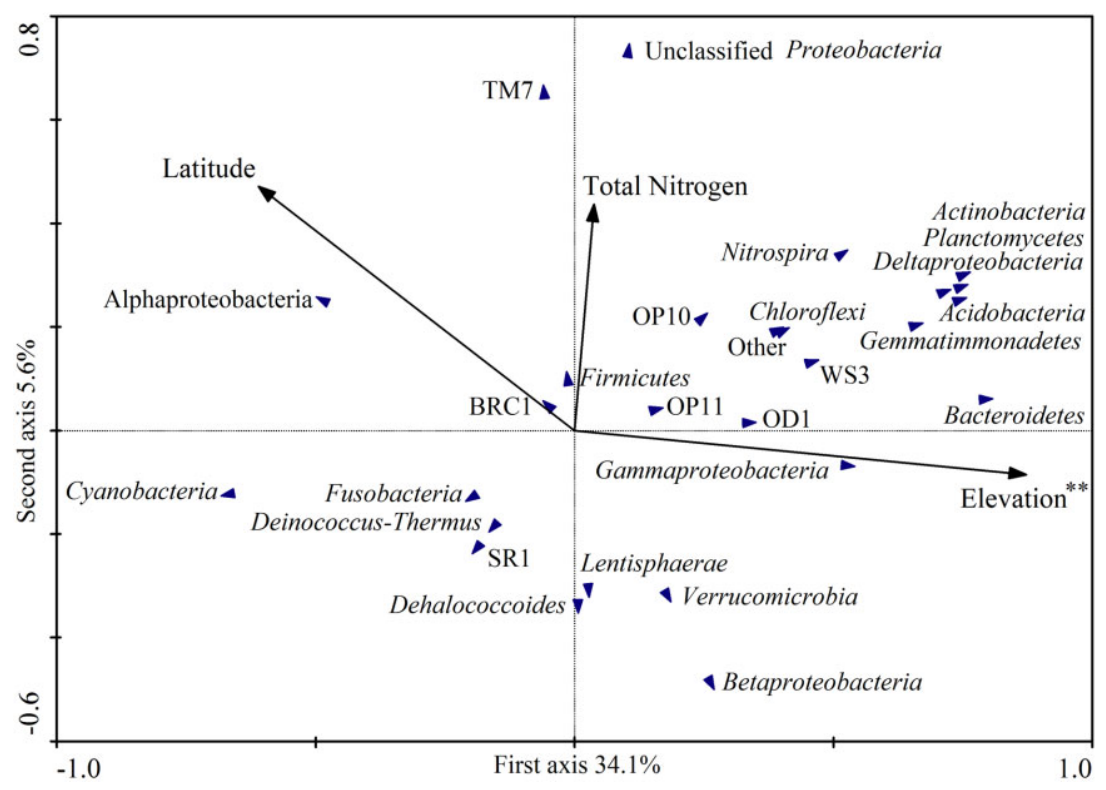

Fig. 2. Redundancy analysis plot for bacterial communities at the phylum level. The environmental variables were automatically selected based on Monte Carlo permutation tests (9999 permutations). Elevation was significant $\left({ }^{* \star} P<0.01\right)$ in explaining the communities and the other two variables (total nitrogen and latitude) were marginally significant. The different phyla were represented by filled triangles that are oriented in different directions. The directions of the triangles and the relative lengths between the triangles and the base point indicate how the phyla linearly responded to the environmental variables. 

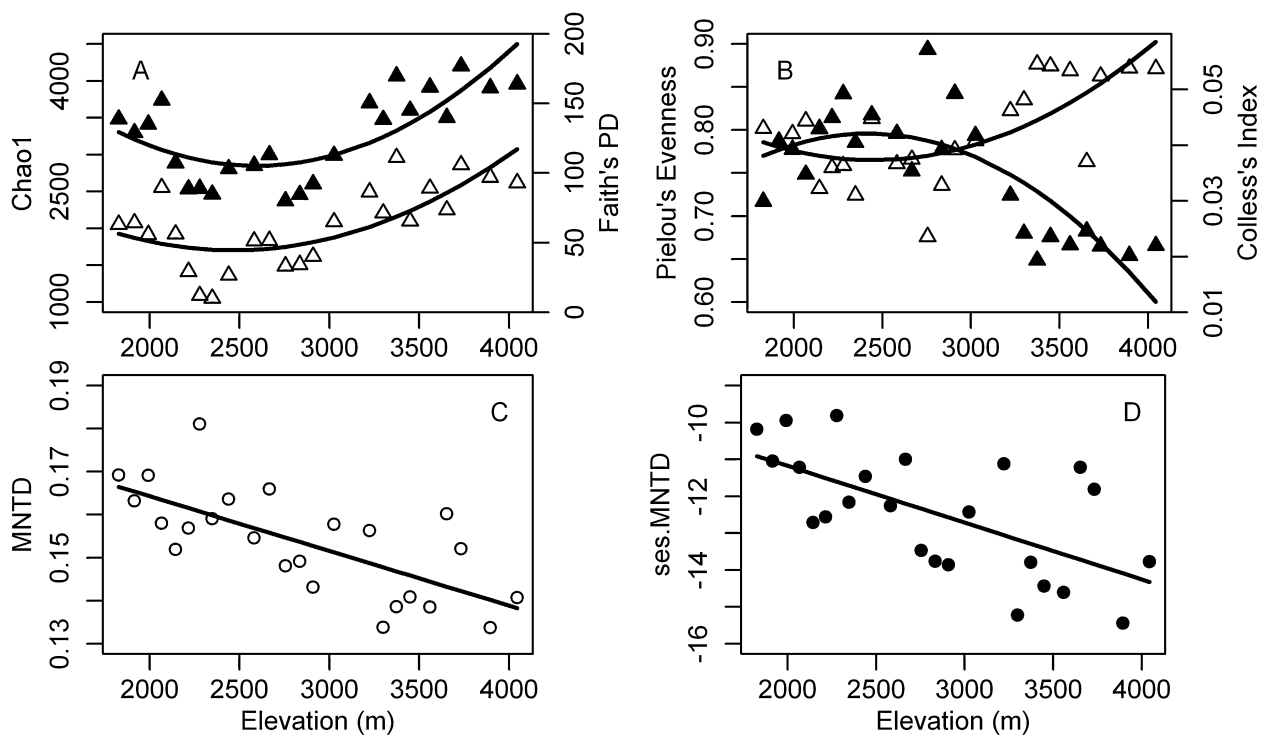

Fig. 3. Biodiversity characteristics of the bacterial communities.

A. Community richness as indicated by Chao1-richness (left $y$-axis, empty triangles) and phylogenetic diversity as indicated by Faith's PD (right $y$-axis, solid triangles) in a hollow pattern along the elevational gradient $\left(r^{2}=0.51, P<0.001\right.$ and $r^{2}=0.58, P<0.001$ respectively). B. Community equitability as indicated by Pielou's evenness (left $y$-axis, empty triangles) in a hollow pattern $\left(r^{2}=0.49, P<0.001\right)$ and phylogenetic tree imbalance as indicated by Colless's index (right $y$-axis, solid triangles) in a unimodal pattern $\left(r^{2}=0.62, P<0.001\right.$ ). C. Observed mean nearest taxon distance (MNTD) in a monotonically decreasing pattern $\left(r^{2}=0.49, P<0.001\right)$ was used to characterize the phylogenetic structure of the bacterial communities.

D. The standardized effect sizes of MNTD (ses.MNTD) in a monotonically decreasing pattern $\left(r^{2}=0.40, P<0.001\right)$ for entire bacterial communities. Significant ses.MNTD values were indicated as solid circles $(P<0.05,1000$ null model runs). The solid lines indicate the prediction of diversity as a function of elevation using a linear or quadratic model with the lowest Akaike's information criterion.

whereas MNTD was most strongly correlated with the TN/total phosphorus (TP) ratio (Table 1).

\section{Discussion}

Analyses of phylogenetic relatedness may provide insights regarding the mechanisms that shape the local communities, such as environmental filtering and competition (Webb et al., 2002; Kembel and Hubbell, 2006; Cavender-Bares et al., 2009). In this study, we examined the phylogenetic structure in stream microbial communities along an extended elevational gradient and found clear elevational patterns for phylogenetic structure, taxonomic richness, phylogenetic diversity, community evenness and community phylogenetic tree imbalance.

We found clear evidence that whole bacterial communities and Proteobacteria alone showed higher terminal phylogenetic clustering at higher elevations than at lower elevations, and this result was independent of the standardization of the MNTD metric.

Our finding regarding the higher phylogenetic clustering at higher elevations at least partly contrasts with the former reports on phylogenetic structure for hummingbird (Graham et al., 2009) and ant (Machac et al., 2010) communities (i.e. the studies that have found strong overdispersion at low elevations), or especially with the finding of increasing phylogenetic overdispersion for angiosperms with elevation (Bryant et al., 2008). The latter study also documented a weak but non-significant increase in phylogenetic structure towards higher elevations for soil Acidobacteria (Bryant et al., 2008). One possible reason for the contradicting results between the two corresponding studies on microbes could be that the elevational gradient considered here might have been more substantial to delineate stronger patterns.

To study the environmental correlates of phylogenetic structure, we related MNTD to measured environmental variables. Overall, phylogenetic structure was highly correlated with the environmental variables $\left(r^{2}=0.751\right.$ for bacteria and $r^{2}=0.686$ for Proteobacteria) (Table 1). This finding is consistent with the significant values of ses.MNTD for all of the elevations, indicating that environmental filtering was a potential primary determinant affecting the community assembly of bacteria. This is also consistent with the previous studies on the same subject (Bryant et al., 2008; Barberán and Casamayor, 2010; Jones and Hallin, 2010), which showed that microbial communities tended to be phylogenetically clustered. It should be noted that this reasoning is based on the view that microbial niches are phylogenetically conserved this assumption still need to be tested for microbes. Based on the observational sampling design used here, 

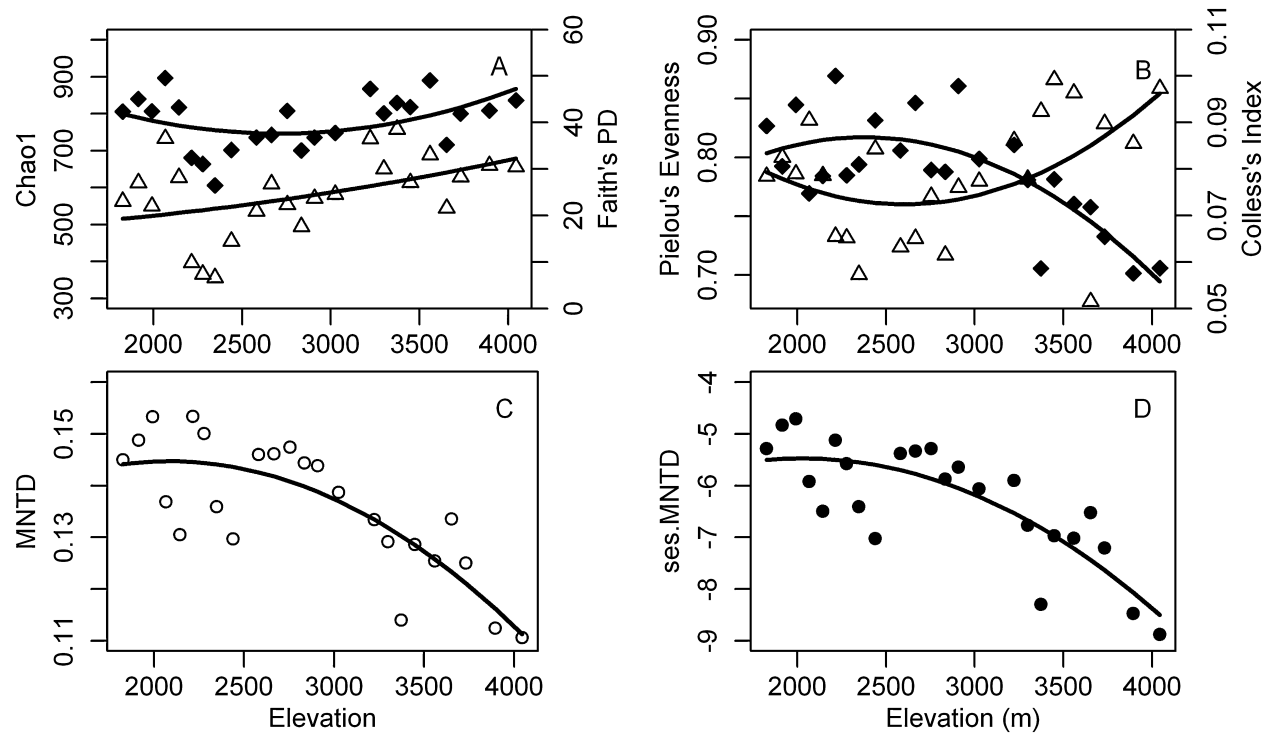

Fig. 4. Biodiversity characteristics for Proteobacteria.

A. Community richness as indicated by Chao1-richness (left $y$-axis, empty triangles) and phylogenetic diversity indicated by Faith's PD (right $y$-axis, solid triangles), which were best described by a hollow-shaped pattern $\left(r^{2}=0.20, P<0.05\right.$ and $r^{2}=0.18, P<0.05$ respectively).

B. Community equitability as indicated by Pielou's evenness (left $y$-axis, empty triangles) with a hollow-shaped pattern $\left(r^{2}=0.24, P<0.01\right)$ and phylogenetic tree imbalance as indicated by Colless's index (right $y$-axis, solid triangles) with a unimodal pattern $\left(r^{2}=0.58, P<0.05\right.$ ).

C. Mean nearest taxon distance (MNTD) in a unimodal pattern $\left(r^{2}=0.46, P<0.001\right)$ was used to characterize the phylogenetic structure of the proteobacterial communities.

D. The standardized effect sizes of MNTD (ses.MNTD) were in a hollow-shaped pattern $\left(r^{2}=0.66, P<0.001\right)$. Significant ses.MNTD values were indicated as solid circles $(P<0.05,1000$ null model runs). The solid lines indicate the prediction of diversity as a function of elevation using a linear or quadratic model with the lowest Akaike's information criterion.

we cannot completely eliminate the possibility that ecological diversification of closely related species also contributed to phylogenetic clustering in these data (Losos, 2008). The phylogenetic and spatial scale of the analyses may also affect the patterns that we documented (Losos, 2008; Cavender-Bares et al., 2009).
However, based on the significant ses.MNTD values and the high correlation between the environmental variables and MNTD, it is unlikely that other processes, such as evolution or species interactions, overwhelm the ecological processes that structure the bacterial communities along the gradient.

Table 1. Relationships between the microbial diversity and potential explanatory variables that were modelled using multiple ordinary least squares regression. The best models were identified using Akaike's information criterion (AIC). The spatial autocorrelation in the model residuals was considered. All of the variables were displayed with increasing $P$-values.

\begin{tabular}{|c|c|c|c|c|c|c|c|c|c|}
\hline \multirow[b]{2}{*}{ Bacteria } & \multirow[b]{2}{*}{ Chao1 } & \multirow[t]{2}{*}{$r^{2}$} & \multirow[t]{2}{*}{ AIC } & \multicolumn{6}{|c|}{ Explanatory variables and $\beta$-weights } \\
\hline & & & & Temp ${ }^{\star * *}$ & $\mathrm{cDOM}^{\star * *}$ & Width ${ }^{* * *}$ & $P C 1^{\star *}$ & Velocity** & $\mathrm{Chl} a^{*}$ \\
\hline & & 0.869 & 43.89 & $-1.074^{a}$ & 0.715 & 0.569 & 0.613 & -0.354 & -0.282 \\
\hline & PD & & & Temp ${ }^{\star \star \star}$ & $\mathrm{CDOM}^{\star \star *}$ & Width" & $P C 1^{* *}$ & $\mathrm{Chl} a^{* *}$ & Size $^{\star}$ \\
\hline & & 0.865 & 44.64 & -1.187 & 1.100 & 0.711 & 0.568 & -0.387 & -0.380 \\
\hline & MNTD & & & Temp ${ }^{\star * *}$ & $\mathrm{TP}^{* \star *}$ & $\mathrm{PC}^{*}$ & Shading & & \\
\hline \multirow{7}{*}{ Proteobacteria } & & 0.751 & 50.67 & 0.708 & 0.551 & -0.377 & 0.259 & & \\
\hline & Chao1 & & & Temp ${ }^{\star * *}$ & Width** & $\mathrm{PC}^{*}$ & $\mathrm{Chl} a^{*}$ & & \\
\hline & & 0.646 & 59.09 & -1.271 & 0.807 & 0.389 & -0.389 & & \\
\hline & PD & & & Temp*** & Width"*** & $\mathrm{Chl} a^{\star *}$ & $\mathrm{PC} 2^{\star \star}$ & & \\
\hline & & 0.680 & 56.70 & -1.172 & 0.885 & -0.504 & 0.450 & & \\
\hline & MNTD & & & $\mathrm{TN} / \mathrm{TP} \mathrm{P}^{\star \star *}$ & Depth** & Width* & & & \\
\hline & & 0.686 & 52.61 & -0.592 & 0.445 & 0.345 & & & \\
\hline
\end{tabular}

a. Standardized partial regression coefficients.

*** $P<0.001 ;{ }^{* *} P<0.01 ;{ }^{*} P<0.05$.

PC1 and PC2, the first and second principal component of the 27 geochemical variables (see text for details); TN/TP: the ratio between total nitrogen (TN) and total phosphorus (TP); cDOM, chromophoric dissolved organic matter; Depth, stream water depth; Width, stream width; Velocity, stream water velocity; Size, the median for substratum particle size; Temp, stream water temperature; CR, community respiration; Shading, riparian shading; $\mathrm{Chl} a$, Chlorophyll $a$. 
The possibility of strong environmental filtering was also reflected in the clear relative-abundance distributions of many of the detected phyla along the elevation. The RDA and abundance plots revealed that the abundance of most of the phyla (e.g. Bacteroidetes and Actinobacteria) increased with elevation, and water temperature, which was significantly linearly related to elevation $(P=0.004)$, explained $17.6 \%$ of the total variation of the composition of the phyla. This indicated that at least some of the taxa may tolerate the harsh, cold environments that are present at higher elevations, and it is consistent with earlier findings. For example, Bacteroidetes were dominant in the moraine lakes and meltwaters on Mount Everest (Liu et al., 2006), and Actinobacteria were dominant in permafrost soils (Rodrigues and Tiedje, 2008) or snow packs (Amato et al., 2007). Temperature was also shown to control bacterial communities in aquatic ecosystems (Hall et al., 2008), such as arctic lakes/streams (Adams et al., 2010), an alkaline hot spring in Yellowstone National Park (Miller et al., 2009) and bacterial communities that are associated with the cyanobacterium Microcystis sp. (Dziallas and Grossart, 2011). These results are also consistent with the idea that bacterial phyla may be divided into ecologically meaningful categories that have distinct roles in the ecosystem (Fierer et al., 2007). The succession of bacterial phyla along the elevational gradient may imply that the occurrence of species with specific traits for adapting to specific habitats (for example, cold environments) may influence phylogenetic clustering at high elevations.

Furthermore, the correlation analyses indicated that water temperature was the strongest environmental filter for phylogenetic structure. For instance, it significantly $(P<0.001)$ explained $51.6 \%$ of the variation in MNTD when the spatial effects were controlled for. This result agrees with Machac and colleagues (2010), who emphasized the importance of temperature in explaining phylogenetic structures in ant communities. Other factors, such as extreme oligotrophy and UV exposure at high elevations, may also affect phylogenetic structure. However, our current results suggested that the increased terminal clustering at high elevations may have been related to low average temperature and relatively high day-night variation in temperature indicating harsh conditions and also to frequent physicochemical disturbances prevailing in upper parts of this stream (Wang et al., 2011). This wide spatiotemporal variability in environmental conditions is typical for small streams (Allan and Castillo, 2007), and especially for those at higher elevations with high daynight temperature variations (Wang et al., 2011). This finding is consistent with a view suggesting that ecosystem disturbances in aquatic environments can result in assemblages that share many closely related species (Helmus et al., 2010), and is in line with an observation that environmental instability facilitates phylogenetic clustering for bacterial communities (Amaral-Zettler et al., 2011). Environmental disturbance may also result in higher evenness in taxonomic communities or a lower imbalance in phylogenetic trees at higher elevations (Figs $2 \mathrm{~B}$ and $4 \mathrm{~B}$ ). This is because potentially competitively superior species may not have enough time to increase their abundance and dominate the community.

In addition to the patterns in phylogenetic structure, we also observed interesting patterns of taxonomic richness and phylogenetic diversity. When we compared the taxonomic richness and phylogenetic diversity for whole bacterial communities or Proteobacteria alone, we observed relatively similar patterns along the elevation, which showed high intercorrelation. This result is not surprising because taxonomic and phylogenetic diversity are typically related (Morlon et al., 2010). Compared with the results of the fingerprinting method (DGGE) for the same samples (Wang et al., 2011), results from pyrosequencing showed slightly different elevational patterns in taxonomic richness. Pyrosequencing detected higher richness at the lowest elevations than at intermediate elevations, whereas DGGE showed a monotonic increase of richness with elevation. Nevertheless, elevation strongly and positively correlated with taxonomic richness for both methods, and the elevational pattern in taxonomic richness was different from the other two organism groups (macroinvertebrates and diatoms) sampled along the same elevational gradient (Wang et al., 2011). This hollow pattern for aquatic bacteria was also different from recent observations for species richness or phylogenetic diversity of terrestrial microbes on mountainsides, such as the decreasing pattern for soil Acidobacteria (Bryant et al., 2008), unimodal pattern for soil bacteria (Singh et al., 2011), and non-significant elevational pattern for soil bacteria or leaf bacteria (Fierer et al., 2011). Therefore, the generality of these observations for microbes still needs to be addressed by more extensive studies for specific habitats, as well as across habitats.

In summary, the hollow elevational pattern in species richness and phylogenetic diversity we detected has rarely been observed in nature (Rahbek, 2005). Bacterial communities showed higher terminal phylogenetic clustering at high elevations than at low elevations, which may have been related to the increased environmental filtering towards high elevations. This filtering may be attributed to the low overall temperature and frequent disturbances that exist at high elevations (e.g. the high day-night temperature variation). Because different phyla or species may respond differently to specific environmental stresses, the environmental conditions created an orderly succession in the relative abundance of different phyla along the elevational gradient. An analysis of the phylogenetic structure revealed that environmental filtering (in this case, temperature) is likely responsible for 
the observed biodiversity patterns. These results further suggest that microorganisms may be more strongly driven by environmental filtering than macroorganisms, which are also structured by species interactions and evolutionary processes.

\section{Experimental procedures}

\section{Field sampling and physicochemical analyses}

The detailed sampling scheme and physicochemical/ biological analyses were previously described by Wang and colleagues (2011), and the methods are briefly described here. In October-November 2009, we picked 26 sampling sites at approximately every $89 \mathrm{~m}$ change in elevation along a stony stream located in Laojun Mountain, Yunnan province, China. The sampling sites extended from 1820 to $4050 \mathrm{~m}$ in elevation. Biofilm was scraped off the stones to obtain subsamples from a predefined area $\left(9 \mathrm{~cm}^{2}\right)$ using a sterilized sponge. Ten subsamples were subsequently pooled into a composite sample from each site. The samples for bacteria were frozen at $-18^{\circ} \mathrm{C}$ immediately after sampling. As failing to obtain the molecular analyses for the microbial communities on two of the biofilm samples, we only analysed 24 samples in this study. More than 50 physicochemical characteristics were measured in situ or in the lab (see Wang et al., 2011 for details). Biofilm characteristics, such as the net photosynthesis production (NPP), biofilm community respiration (CR) and chlorophyll a, were measured as previously described (Wang et al., 2011).

\section{DNA extraction, amplification and sequencing}

Genomic DNA was extracted from freeze-dried biofilm using the phenol chloroform method as described by Zhou and colleagues (1996). Genomic DNA was then concentrated to a volume of $100 \mu \mathrm{l}$. Bacterial 16S rRNA genes were amplified using the $27 \mathrm{~F}$ primer with the 454 Life Sciences ' $A$ ' sequencing adapter, and the modified 519R primer with a 8 bp barcode sequence and the 454 Life Sciences 'B' sequencing adapter (Hamady et al., 2008). Three replicates of PCR amplifications were performed per sample. The PCR products were checked by electrophoresis, and replicates were combined. The purified, barcoded amplicons were pooled at equimolar ratios at a final concentration of $100 \mathrm{ng}^{-1}$, and then sequenced using a Roche 454 FLX pyrosequencer.

\section{Analysis of pyrosequenced amplicons}

The sequences that were generated by pyrosequencing the bacterial 16S rRNA gene amplicons were processed using the QIIME pipeline (the Quantitative Insights into
Microbial Ecology, v1.2) (Caporaso et al., 2010b). In brief, sequences that were longer than 200 bp were denoised with the Denoiser algorithm (Reeder and Knight, 2010), clustered into OTUs at $97 \%$ pairwise identity with the seed-based uclust algorithm (Edgar, 2010). After chimeras were removed via Chimera Slayer (Haas et al., 2011), representative sequences from each OTU were aligned to the Greengenes imputed core reference alignment (DeSantis et al., 2006) using PyNAST (Caporaso et al., 2010a). After removing gaps and hypervariable regions using a Lane mask, the alignments were then used to construct an approximate maximum-likelihood tree using RAxML v7.0.3 (Stamatakis, 2006). The taxonomic identity of each representative sequence was determined using the RDP Classifier (Wang et al., 2007).

In total, we obtained 170698 quality sequences for all of the sites, which ranged from 4694 to 10672 per sample with an average length of approximately $400 \mathrm{bp}$. Based on the OTU identification, a community data matrix that contained 14071 unique OTUs was constructed. For each calculated metrics, we accounted for the differences in the sampling efforts among the samples by randomly subsampling 4000 sequences per sample for 1000 times. Because the sequence number for Proteobacteria was the highest among the phyla for 21 sites and the numbers for the other phyla were small for some of the sites, we considered also Proteobacteria separately in the following biodiversity analyses. For the specific phylum Proteobacteria, we subsampled the community data matrix while excluding sequences of the other phyla, and then randomly subsampled 1400 sequences per sample for 1000 times.

\section{Biodiversity analyses}

Chao1-richness (Chao, 1984) and Pielou's evenness (Pielou, 1966) were used to estimate the community diversity. Chao1-richness is a non-parametric estimator of richness (Chao, 1984) that is computed as Chao1richness $=S_{\text {obs }}+\left[a^{2} /(2 \times b)\right]$, where $S_{\text {obs }}$ is the number of species observed, and $a$ or $b$ are the number of species that are observed just once or twice. Further, we included Faith's PD (Faith, 1992) and Colless's index (Colless, 1982 ) to estimate the phylogenetic community diversity. Faith's PD measures the total phylogenetic branch length that joins the basal node to the tips of all the species in the sample (Faith, 1992). Colless's index measures phylogenetic tree imbalance as the sum of absolute values $|L-R|$ at each node of the tree, where $L$ (or R) is the size of the left (or right) daughter clade at the node (Colless, 1982); it was further normalized by the number of pairwise treetip combinations [i.e. by dividing (tip number -1$) \times($ tip number - 2)/2].

For the phylogenetic community structure, we calculated the MNTD of all of the species pairs occurring in a 
community based on the observed community dataset (Webb et al., 2002). Mean nearest taxon distance is an estimate of the mean phylogenetic relatedness between each OTU in a bacterial community and its nearest relative. To compensate for random processes in the observed phylogenetic community structure along the studied elevational gradient, we further calculated the differences in the phylogenetic distances between the observed and randomly generated null communities, and we standardized them using the standardized deviation of phylogenetic distances in 1000 null communities (Webb, 2000). We generated these null communities with the assumption that all species that exist along the elevation are equally able to colonize any elevation without dispersal limitation at local spatial scales, and thus each species has the same expected prevalence (Gotelli, 2000; Kembel and Hubbell, 2006; Helmus et al., 2007). The total species richness of each elevation was kept standard, and species at each elevation were chosen randomly without replacement from the pool of species present along the elevation. The obtained standardized effect size measure (ses.MNTD) can be used to test for phylogenetic clustering or overdispersion (Webb, 2000). Negative ses.MNTD values and low quantiles $(P<0.05)$ indicate that co-occurring species are more closely related than expected by chance (clustering), whereas positive values and high quantiles $(P>0.95)$ indicate that the co-occurring species are less closely related than expected by chance (overdispersion) (Webb, 2000). These analyses were implemented in the $R$ environment (http://www.r-project.org) with the package Picante 1.2-0 (Kembel et al., 2010).

\section{Statistical analyses}

We used the RDA to examine the potential explanatory variables for the community composition at the phylum level. This is because the length of the first detrended correspondence analysis axis that was performed on the phylum data was lower than 2, which indicate linear responses of the taxa to environmental gradients. By performing a principal component analysis (PCA), the electronic conductivity, alkalinity and dissolved ions $\left(\mathrm{Si}, \mathrm{Cl}^{-}\right.$, $\mathrm{SO}_{4}{ }^{2-}, \mathrm{K}, \mathrm{Na}, \mathrm{Ca}, \mathrm{Mg}, \mathrm{Ba}, \mathrm{Sr}, \mathrm{As}, \mathrm{Al}, \mathrm{Fe}, \mathrm{Mn}, \mathrm{Zn}, \mathrm{Cr}, \mathrm{Cu}$, $\mathrm{Pb}, \mathrm{Ni}, \mathrm{PO}_{4}{ }^{3-}, \mathrm{NH}_{4}{ }^{+}, \mathrm{NO}_{2}{ }^{-}, \mathrm{NO}_{3}{ }^{-}, \mathrm{DIC}, \mathrm{HCO}_{3}{ }^{-}$and $\mathrm{CO}_{3}{ }^{2-}$ ) were reduced to the first two principal components (PC1 and PC2) as explanatory variables that represent environmental factors (Wang et al., 2011). This process was performed to decrease the degrees of freedom such that they were lower than the number of sampled sites. The remaining measured variables (i.e. water temperature, $\mathrm{pH}$, chlorophyll a, NPP, CR, riparian shading, stream width, water depth, water velocity, median for substratum particle size, chromophoric dissolved organic matter, dissolved organic carbon, TN, TP and the molecular ratio of TN to TP) were used as environmental variables without a PCA step (Wang et al., 2011). All of the significant environmental variables, as well as elevation, longitude and latitude, were selected by forward selection against the Hellingertransformed abundance phylum data with 9999 permutations. These analyses were performed with the software CANOCO 4.53 and the R environment (http://www.rproject.org).

To correlate the observed biodiversity patterns with the environmental variables, we used multiple OLS regression. All of the environmental variables and biodiversity metrics were standardized at a mean of 0 and a standard deviation of 1. Akaike's information criterion was used to identify the most parsimonious model (Fotheringham et al., 2002), and the spatial autocorrelation was taken into account by including eigenvector-based spatial filters that were derived from the geographic distances in all of the models (Diniz-Filho and Bini, 2005). The regression analyses were performed using the software SAM 4.0 (Spatial Analysis in Macroecology, http://www.ecoevol. ufg.br/sam) (Rangel et al., 2010).

\section{Acknowledgements}

We are grateful to Yong Zhang for helping in the field sampling. J. Shen and J.J. Wang were supported by the National Basic Research Program of China (2012CB956100), NSFC (40903031), Jiangsu NSF (BK2010605), and the China Postdoctoral Science Foundation (2011 M500397). J. Soininen was supported by Academy of Finland (126718), University of Helsinki and State Key Laboratory of Lake Science and Environment.

\section{References}

Adams, H.E., Crump, B.C., and Kling, G.W. (2010) Temperature controls on aquatic bacterial production and community dynamics in arctic lakes and streams. Environ Microbiol 12: 1319-1333.

Allan, D.J., and Castillo, M.M. (2007) Stream Ecology: Structure and Function of Running Waters. Dordrecht, The Netherlands: Springer Verlag.

Amaral-Zettler, L.A., Zettler, E.R., Theroux, S.M., Palacios, C., Aguilera, A., and Amils, R. (2011) Microbial community structure across the tree of life in the extreme Rio Tinto. ISME J 5: 42-50.

Amato, P., Hennebelle, R., Magand, O., Sancelme, M., Delort, A.M., Barbante, C., et al. (2007) Bacterial characterization of the snow cover at Spitzberg, Svalbard. FEMS Microbiol Ecol 59: 255-264.

Auguet, J.-C., Barberan, A., and Casamayor, E.O. (2010) Global ecological patterns in uncultured Archaea. ISME $J$ 4: 182-190.

Barberán, A., and Casamayor, E. (2010) Global phylogenetic community structure and $\beta$-diversity patterns in surface 
bacterioplankton metacommunities. Aquat Microb Ecol 59: $1-10$.

Bohannan, B. (2003) New approaches to analyzing microbial biodiversity data. Curr Opin Microbiol 6: 282-287.

Bryant, J.A., Lamanna, C., Morlon, H., Kerkhoff, A.J., Enquist, B.J., and Green, J.L. (2008) Microbes on mountainsides: contrasting elevational patterns of bacterial and plant diversity. Proc Natl Acad Sci USA 105: 11505-11511.

Cadotte, M.W., Hamilton, M.A., and Murray, B.R. (2009) Phylogenetic relatedness and plant invader success across two spatial scales. Divers Distrib 15: 481-488.

Caporaso, J.G., Bittinger, K., Bushman, F.D., DeSantis, T.Z., Andersen, G.L., and Knight, R. (2010a) PyNAST: a flexible tool for aligning sequences to a template alignment. Bioinformatics 26: 266-267.

Caporaso, J.G., Kuczynski, J., Stombaugh, J., Bittinger, K., Bushman, F.D., Costello, E.K., et al. (2010b) QIIME allows analysis of high-throughput community sequencing data. Nat Meth 7: 335-336.

Cavender-Bares, J., Kozak, K.H., Fine, P.V.A., and Kembel, S.W. (2009) The merging of community ecology and phylogenetic biology. Ecol Lett 12: 693-715.

Chao, A. (1984) Nonparametric estimation of the number of classes in a population. Scand J Stat 11: 265-270.

Colless, D.H. (1982) Phylogenetics: the theory and practice of phylogenetic systematics. Syst Zool 31: 100-104.

DeSantis, T.Z., Hugenholtz, P., Larsen, N., Rojas, M., Brodie, E.L., Keller, K., et al. (2006) Greengenes, a chimerachecked 16S rRNA gene database and workbench compatible with ARB. Appl Environ Microbiol 72: 50695072.

Diniz-Filho, J.A.F., and Bini, L.M. (2005) Modelling geographical patterns in species richness using eigenvectorbased spatial filters. Glob Ecol Biogeogr 14: 177-185.

Downing, A.L., and Leibold, M.A. (2002) Ecosystem consequences of species richness and composition in pond food webs. Nature 416: 837-841.

Dziallas, C., and Grossart, H.-P. (2011) Temperature and biotic factors influence bacterial communities associated with the cyanobacterium Microcystis sp. Environ Microbiol 13: 1632-1641.

Edgar, R.C. (2010) Search and clustering orders of magnitude faster than BLAST. Bioinformatics 26: 24602461.

Emerson, B.C., and Gillespie, R.G. (2008) Phylogenetic analysis of community assembly and structure over space and time. Trends Ecol Evol 23: 619-630.

Faith, D.P. (1992) Conservation evaluation and phylogenetic diversity. Biol Conserv 61: 1-10.

Fierer, N., Bradford, M.A., and Jackson, R.B. (2007) Toward an ecological classification of soil bacteria. Ecology 88: 1354-1364.

Fierer, N., McCain, C., Meir, P., Zimmerman, M., Rapp, J., Silman, M., and Knight, R. (2011) Microbes do not follow the elevational diversity patterns of plants and animals. Ecology 92: 797-804.

Fotheringham, A.S., Brunsdon, C., and Charlton, M. (2002) Geographically Weighted Regression: The Analysis of Spatially Varying Relationships. Chichester, UK: Wiley.

Gotelli, N.J. (2000) Null model analysis of species co-occurrence patterns. Ecology 81: 2606-2621.
Graham, C.H., Parra, J.L., Rahbek, C., and McGuire, J.A. (2009) Phylogenetic structure in tropical hummingbird communities. Proc Natl Acad Sci USA 106: 19673-19678.

Grytnes, J., and McCain, C. (2007) Elevational trends in biodiversity. In Encyclopedia of Biodiversity. Levin, S. (ed.). Amsterdam, The Netherlands: Elsevier, pp. 1-8.

Haas, B.J., Gevers, D., Earl, A.M., Feldgarden, M., Ward, D.V., Giannoukos, G., et al. (2011) Chimeric 16S rRNA sequence formation and detection in Sanger and 454pyrosequenced PCR amplicons. Genome Res 21: 494504.

Hall, E.K., Neuhauser, C., and Cotner, J.B. (2008) Toward a mechanistic understanding of how natural bacterial communities respond to changes in temperature in aquatic ecosystems. ISME J 2: 471-481.

Hamady, M., Walker, J.J., Harris, J.K., Gold, N.J., and Knight, R. (2008) Error-correcting barcoded primers for pyrosequencing hundreds of samples in multiplex. Nat Meth 5: 235-237.

Helmus, M.R., Bland, T.J., Williams, C.K., and Ives, A.R. (2007) Phylogenetic measures of biodiversity. Am Nat 169: 68-83.

Helmus, M.R., Keller, W., Paterson, M.J., Yan, N.D., Cannon, C.H., and Rusak, J.A. (2010) Communities contain closely related species during ecosystem disturbance. Ecol Lett 13: 162-174.

Horner-Devine, C.M., and Bohannan, B.J.M. (2006) Phylogenetic clustering and overdispersion in bacterial communities. Ecology 87: 100-108.

Jones, C.M., and Hallin, S. (2010) Ecological and evolutionary factors underlying global and local assembly of denitrifier communities. ISME J 4: 633-641.

Kembel, S.W. (2009) Disentangling niche and neutral influences on community assembly: assessing the performance of community phylogenetic structure tests. Ecol Lett 12: 949-960.

Kembel, S.W., and Hubbell, S.P. (2006) The phylogenetic structure of a neotropical forest tree community. Ecology 87: 86-99.

Kembel, S.W., Cowan, P.D., Helmus, M.R., Cornwell, W.K., Morlon, H., Ackerly, D.D., et al. (2010) Picante: R tools for integrating phylogenies and ecology. Bioinformatics 26: 1463-1464.

Kluge, J., and Kessler, M. (2010) Phylogenetic diversity, trait diversity and niches: species assembly of ferns along a tropical elevational gradient. J Biogeogr 38: 394-405.

Liu, Y., Yao, T., Jiao, N., Kang, S., Zeng, Y., and Huang, S. (2006) Microbial community structure in moraine lakes and glacial meltwaters, Mount Everest. FEMS Microbiol Lett 265: 98-105.

Lomolino, M.V. (2001) Elevation gradients of species-density: historical and prospective views. Glob Ecol Biogeogr 10: 3-13.

Losos, J.B. (2008) Phylogenetic niche conservatism, phylogenetic signal and the relationship between phylogenetic relatedness and ecological similarity among species. Ecol Lett 11: 995-1003.

Machac, A., Janda, M., Dunn, R.R., and Sanders, N.J. (2010) Elevational gradients in phylogenetic structure of ant communities reveal the interplay of biotic and abiotic constraints on diversity. Ecography 34: 364-371. 
Martin, A.P. (2002) Phylogenetic approaches for describing and comparing the diversity of microbial communities. Appl Environ Microbiol 68: 3673-3682.

Miller, S.R., Strong, A.L., Jones, K.L., and Ungerer, M.C. (2009) Bar-coded pyrosequencing reveals shared bacterial community properties along the temperature gradients of two alkaline hot springs in Yellowstone National Park. Appl Environ Microbiol 75: 4565-4572.

Morlon, H., Schwilk, D., Bryant, J., Marquet, P., Rebelo, A., Tauss, C., et al. (2010) Spatial patterns of phylogenetic diversity. Ecol Lett 14: 141-149.

Pielou, E. (1966) The measurement of diversity in different types of biological collections. J Theor Biol 13: 131-144.

Rahbek, C. (2005) The role of spatial scale and the perception of large-scale species-richness patterns. Ecol Lett 8: 224-239.

Rangel, T.F., Diniz-Filho, J.A.F., and Bini, L.M. (2010) SAM: a comprehensive application for Spatial Analysis in Macroecology. Ecography 33: 46-50.

Reche, I., Pulido-Villena, E., Morales-Baquero, R., and Casamayor, E.O. (2005) Does ecosystem size determine aquatic bacterial richness? Ecology 86: 1715-1722.

Reeder, J., and Knight, R. (2010) Rapidly denoising pyrosequencing amplicon reads by exploiting rank-abundance distributions. Nat Meth 7: 668-669.

Rodrigues, D.F., and Tiedje, J.M. (2008) Coping with Our Cold Planet. Appl Environ Microbiol 74: 1677-1686.

Singh, D., Takahashi, K., Kim, M., Chun, J., and Adams, J.M. (2011) A Hump-Backed Trend in Bacterial Diversity with Elevation on Mount Fuji, Japan. Microb Ecol. doi: 10.1007/s00248-00011-09900-00241.

Stamatakis, A. (2006) RAxML-VI-HPC: maximum likelihoodbased phylogenetic analyses with thousands of taxa and mixed models. Bioinformatics 22: 2688-2690.

Vamosi, S.M., and Queenborough, S.A. (2010) Breeding systems and phylogenetic diversity of seed plants along a large-scale elevational gradient. J Biogeogr 37: 465-476.

Vamosi, S.M., Heard, S.B., Vamosi, J.C., and Webb, C.O. (2009) Emerging patterns in the comparative analysis of phylogenetic community structure. Mol Ecol 18: 572592.

Wang, J., Soininen, J., Zhang, Y., Wang, B., Yang, X., and Shen, J. (2011) Contrasting patterns in elevational diversity between microorganisms and macroorganisms. J Biogeogr 38: 595-603.

Wang, Q., Garrity, G.M., Tiedje, J.M., and Cole, J.R. (2007) Naive Bayesian classifier for rapid assignment of rRNA sequences into the new bacterial taxonomy. Appl Environ Microbiol 73: 5261-5267.

Webb, C.O. (2000) Exploring the phylogenetic structure of ecological communities: an example for rain forest trees. Am Nat 156: 145-155.

Webb, C.O., Ackerly, D.D., McPeek, M.A., and Donoghue, M.J. (2002) Phylogenies and community ecology. Annu Rev Ecol Syst 33: 475-505.

Zhou, J., Bruns, M.A., and Tiedje, J.M. (1996) DNA recovery from soils of diverse composition. Appl Environ Microbiol 62: 316-322. 